\title{
CONVENIO 169 Y SU IMPLEMENTACIÓN. LA PROPUESTA DEL GOBIERNO BACHELET (2006 - 2010)
}

\author{
Rodrigo Egaña B. \\ Universidad de Chile \\ regana@iap.uchile.cl
}

El artículo ofrece una visión general de cómo el Convenio 169 de la OIT sobre pueblos indígenas fue diseñado e implementado por el gobierno de Michelle Bachelet. Se argumenta que si bien el Gobierno busco una forma amplia de aplicación que abarcaba todos los temas considerados en el Convenio, el corto tiempo disponible para alcanzar resultados fue factor clave para impedir el desarrollo de algunas iniciativas.

Palabras clave: Convenio 169, pueblos indigenas, politicas publicas, Chile.

\section{CONVENTION 169 AND ITS IMPLEMENTATION. THE BACHELET GOVERNMENT PROPOSAL (2006-2010)}

This article offers an overview of how the Convention 169 of OIT about indigenous people was designed and implemented by the Bachelet Administration in Chile (2006-2010). It is argued that although government's implementation covered all the aspects considered in this Convention, the short time considered to obtain results was a key factor that weakened the development of this initiatives.

Keyword: Convention 169, indigenous peoples, public policy, Chile. 


\section{El proceso de aprobación del Convenio i69 de la OIT}

\subsection{ANTECEDENTES SOBRE LA SUSCRIPCIÓN DEL CONVENIO}

Es posible sostener que, al momento en que se suscribe por el gobierno de Chile el Convenio 169, que había sido aprobado por la OIT en el año 1989, esta se hace sin haber realizado con anterioridad una discusión profunda sobre las implicancias que tenía adherir al documento y comprometerse como Estado y como sociedad a lo que el convenio estipula.

Lo anterior hay que entenderlo en el contexto histórico en el cual se estaba y lo que significaba pasar de la dictadura militar a un régimen democrático. La demanda indígena que se había ido construyendo en los años ochenta, es casi enteramente recogida en el llamado Acuerdo de Nueva Imperial ${ }^{1}$, en el cual se fija el primer acuerdo político entre el sector que asumiría posteriormente el gobierno y el mundo indígena. Parte del Acuerdo es la suscripción y posterior ratificación y puesta en marcha del Convenio 169. Pero al asumirlo, esto se hacía sin que hubiera una cabal comprensión de lo que implicaba en términos de defensa de los derechos indígenas ni menos lo que debía fluir hacia las tareas de definición e implementación de políticas públicas.

Cabe mencionar que hacia el año 1990, no existía instituido en el país ningún sistema de participación ciudadana ni menos instalado algún sistema de consulta por parte del gobierno en el proceso de formulación de políticas públicas y de proyectos de ley. De ahí que era difícil imaginar qué implicaba la obligación a consulta indígena que forma parte del convenio.

Tampoco había una clara comprensión sobre lo que podía significar que los pueblos indígenas tuvieran un mayor control sobre sus territorios y sus recursos naturales, ni lo que podía significar la validación de normas penales propias.

Al momento de proponer el Presidente Patricio Aylwin al Congreso la ratificación del Convenio 169, surgió la pregunta relativa a las razones que hacían necesaria la ratificación del mismo; cuál era la obligatoriedad de ratificarlo. Podemos afirmar que para el presidente de la época era claro que había que dar una señal política en el sentido de incorporar en el sistema jurídico chileno el reconocimiento de

$1 \quad$ Acuerdo de Nueva Imperial. 1 de diciembre de 1989. 
los pueblos indígenas que contenía el convenio. ${ }^{2}$ Esta convicción la mostró el Ex-Presidente cuando presidió la Comisión redactora del "Informe sobre la verdad histórica y el nuevo trato para los pueblos indígenas” y en su preocupación permanente que ha mostrado sobre esta materia.

\subsection{LA DISCUSIÓN PARLAMENTARIA}

Pero esta convicción no era compartida por el conjunto de la clase política.

Durante la larga discusión parlamentaria, que tomó 17 años, sucesivamente los gobiernos de la Concertación expresaron su compromiso con la ratificación del convenio. Este compromiso fue reflejado tanto en los programas de gobierno como en distintos momentos en cada período gubernamental. Si bien el convenio se envió al parlamento en el año 1991, sólo en el 2000 fue aprobado por la Cámara de Diputados y en el 2008 por el Senado ${ }^{3}$.

La demanda indígena por la ratificación fue tomando fuerza en el gobierno del Presidente Ricardo Lagos, sobre todo a partir del Informe sobre Verdad Histórica y Nuevo Trato, que se preparó durante ese mandato. Esta demanda fue asumida por el gobierno de Michelle Bachelet, la que puso mayor énfasis en tratar de obtener su aprobación.

La discusión habida en el Senado es relevante a tener en cuanta, por las implicancias que tiene para la implementación del convenio una vez aprobado. El Ministro de la Segpres de la época, José Antonio Viera-gallo, sostuvo que las normas contenidas en el convenio ya estaban reflejadas en la normativa indígena vigente $^{4}$ y que lo señalado por el convenio se debía entender como principios orientadores de la acción política sin tener efectos normativos directos. Es decir, el convenio era considerado más bien una declaración programática sin tener el carácter de una norma de carácter ejecutorio. Lo anterior llevó a que, a pesar que el Tribunal Constitucional había ya declarado en el año 2000 que los artículos

2 Ver mensaje enviado al Congreso el 27 de diciembre de 1990 para la ratificación del Convenio y la presentación del Proyecto de Ley Indígena de fecha 15 de octubre de 1991.

3 Hay que tener en consideración que si el convenio era rechazado por cualquiera de las dos cámaras, este quedaba definitivamente rechazado, de acuerdo a las normas constitucionales vigentes.

$4 \quad$ Ley Indígena Nr. 19.253, art. 34. 
6 y 7 del convenio tenían un carácter autoejecutable ${ }^{5}$, el Senado al aprobar el convenio destacó su carácter programático ${ }^{6}$, lo que se traducía en que a partir de la aprobación del mismo no sería necesario realizar cambios en otros cuerpos legales para adecuarlos a los contenidos del convenio.

\subsection{ENTRE LA APROBACIÓN Y LA RATIFICACIÓN DEL CONVENIO}

Con posterioridad a la aprobación en el Senado, se abrió una nueva discusión referida a la forma como la Presidenta debía ratificar, ante la OIT, la adhesión de Chile al convenio.

Los convenios internacionales deben ser aprobados o rechazados por el parlamento en su integralidad, no es posible aprobar algunos artículos y rechazar otros. Lo que sí es posible es que al momento de inscribir la ratificación del país a un cierto convenio, esta se realice manifestando notas aclaratorias por medio de las cuales, a un determinado artículo se le hace una salvedad. Esto ha ocurrido en otros convenios de la OIT. En este sentido, en la comunicación del Senado a la Presidencia se señaló que en relación al art. 35 del convenio, se manifestaba una salvedad. El art. 35 señala que las disposiciones del Convenio no deberán menoscabar los derechos y ventajas garantizados por otros instrumentos internacionales.

Lo que estaba detrás de la propuesta que fue presentada por los senadores de la Alianza y apoyada por el Presidente de la Comisión de RREE del Senado ${ }^{7}$ era la validez o no validez jurídica en la normativa chilena de la declaración de Naciones Unidas sobre el derecho de los pueblos indígenas. Si bien Chile había suscrito dicha declaración, no había intención de ratificarla, sino de asumirla sólo como una orientación programática.

Es en este contexto, que tomó importancia el hecho de si la ratificación que hiciera la Presidenta ante la OIT iba a contener o no esta cláusula interpretativa del art. 35. Consultada la OIT por el Gobierno, ésta contestó que el convenio establece las normas mínimas, las que no deberían menoscabar el goce de normas más favorables que puedan existir a nivel nacional, incluidas aquellas que sean

5 Fallo del Tribunal Constitucional, Rol nr. 309 del 2000 y nr. 1050 del 2008.

6 Ver Aylwin, José, El Mostrador, 24 marzo 2008.

7 Senador Muñoz Parra, miembro del PPD. 
productos de ratificar otros instrumentos ${ }^{8}$. Esto significaba que, según la OIT, el convenio podía ratificarse con una cláusula interpretativa sin afectar el valor jurídico del mismo. Más aún, de acuerdo a la norma constitucional, los convenios internacionales sólo pasan a ser ley de la república una vez que son aprobados y promulgados como tal.

El tema, que por lo señalado no tenía implicancia jurídica significativa, sí tenía una de valor político y simbólico, tanto para los que querían restringir las posibles implicancias del convenio como para la dirigencia indígena.

Para los dirigentes de la Alianza por Chile el incluir la cláusula interpretativa significaba señalar explícitamente que la declaración universal sobre los derechos humanos de los pueblos indígenas no era aplicable en Chile, asunto que por lo demás, dada la norma seńalada para la aprobación de instrumentos internacionales, era obvio.

Pero para la dirigencia indígena y los sectores preocupados por los derechos indígenas, la inclusión de la cláusula interpretativa era desvirtuar el sentido y el contenido del convenio; ciertamente hacerlo agregaba un elemento adicional a la ya conflictiva relación que existía entre esos sectores y el gobierno.

La decisión a ser tomada por la Presidenta de introducir o no una cláusula interpretativa, conflictuaba dos situaciones diferentes; por una parte el poder enrarecer la relación con el Senado o por otra el empeorar la relación con los pueblos indígenas; la primera era de carácter temporal, la segunda podía tener un carácter más permanente, por el hecho de que se interpretaría que con cláusula el convenio era ratificado con restricciones. Frente a este dilema, la Presidenta optó por ratificar el convenio sin ninguna cláusula interpretativa; ${ }^{9}$ en su opinión se estaba jugando una cuestión histórica y no una meramente temporal y sus convicciones la llevaron a ratificar el convenio tal como lo hizo. Finalmente el Convenio se ratificó sin adición alguna el 9-9-2008 y fue promulgado el 14-10-2008.

8 Ver intercambio de cartas entre Segpres y OIT, marzo 2008.

9 Ver texto de decreto promulgatorio del 9-9-2008. 


\subsection{LA PREPARACIÓN DE LA ENTRADA EN VIGENCIA DEL CONVENIO.}

En paralelo al proceso de ratificación formal del Convenio 169, la Presidenta decidió empezar a preparar la implementación del convenio, dado que un conjunto de disposiciones del mismo dependían de la capacidad de gestión del ejecutivo, para lo cual no era necesario tener el convenio ratificado. El instrumento utilizado para iniciar este proceso fue la dictación de un Instructivo Presidencial, que es el instrumento por medio el cual un presidente refleja la voluntad política sobre cómo desea que se implemente un ámbito de la gestión del gobierno. ${ }^{10}$

En el Instructivo Presidencial se reconoce que el convenio fija un compromiso a asumir por el Estado y que el país debe avanzar en la implementación del mismo. Lo central del instructivo dice relación con que la administración debe adecuar su quehacer en los ámbitos que forman parte del convenio, para ajustarlos a los estándares dictaminados por el mismo. Además, el instructivo establece una estrategia para implementar el convenio, la cual se expresa en el documento de política "Reconocer, Pacto social por la multiculturalidad".

Los principales temas tratados e instruidos en el instructivo son los siguientes:

- crear en todos los ministerios e intendencias las unidades de asuntos indígenas.

- promover políticas de reconocimiento, promoción y fomento de la diversidad cultural.

- adecuar las políticas, planes y programas realizados por el gobierno a la pertinencia indígena; se debía revisar los que afectaban directamente al mundo indígena y ajustarlos desde el punto de vista de su pertinencia indígena.

- establecer mecanismos de información y consulta a los pueblos indígenas en el diseño de las medidas administrativas y legislativas que pudieran afectarles. Esto estaba relacionado con la puesta en marcha del mecanismo de consulta que forma parte del convenio, según el cual el gobierno debía consultar y que esta consulta debía cumplir ciertos estándares.

10 Ver Instructivo Presidencial nr. 5 del 25 de junio del 2008. 
- por último, en relación al presupuesto nacional, señalaba que se debía identificar el conjunto de recursos que el gobierno destinaba a beneficiar al mundo indígena, independiente del servicio público que lo ejecutara.

El Convenio 169 entró en vigencia el 15 de septiembre del 2009, después de haber tenido un año de período de latencia desde su ratificación, año destinado a permitir que el país se adecuara a la entrada en vigencia del mismo. Durante este año, se produjo, en abril del 2009, la visita del Alto Comisionado de Naciones Unidas para los Pueblos Indígenas Dr. James Anaya. Preocupación principal del Comisionado Anaya era conocer en el país mismo la forma como el gobierno estaba preparando la entrada en vigencia del convenio.

Al momento de producirse la visita, el Gobierno ya estaba ejecutando el instructivo presidencial de junio del 2008, entre los cuales destacaba la consulta que se estaba realizando sobre un texto de reforma constitucional de reconocimiento de los pueblos indígenas, que estaba discutiendo la Comisión de Constitución, Legislación y Justicia del Senado. Al mismo tiempo, se estaba haciendo otra consulta sobre las propuestas de nueva institucionalidad política que reflejara los derechos de los pueblos indígenas, que habían sido enunciadas en el documento de política Reconocer.

Después de realizar una visita en la cual se entrevistó con diversas autoridades de gobierno y representantes del mundo indígena, el Comisionado Anaya entregó un conjunto de recomendaciones ${ }^{11}$. Estas versaban sobre la necesidad de que la reforma constitucional debía ser consultada de acuerdo a las normas internacionales, sobre la necesidad de que la consulta fuera hecha tanto por el gobierno como por el parlamento, que la consulta debía ser un proceso transparente y debía cumplir los estándares definidos en el convenio 169 (previa, más que sólo información, generar confianza, método adecuado, sistemática y transparente), señalando además un conjunto de aspectos administrativos necesarios de tomar en consideración (difusión, organización, ámbitos temáticos, geográficos y temporales). En síntesis, el Gobierno se preparaba para la entrada en vigencia del Convenio 169, tanto a nivel de la política indígena, a nivel de la consulta y a nivel de ajustes normativos.

11 Ver Informe del Comisionado Dr. James Anaya sobre visita a Chile de abril 2009. 


\section{El contexto político para la puesta en Vigencia del Convenio i69}

\subsection{LA SITUACIÓN AL MOMENTO DE LA APROBACIÓN POR EL PARLAMENTO}

Al momento de la aprobación del convenio, marzo del 2008, en el país se observaba una situación bastante conflictiva en el mundo indígena, que había llevado a que la Presidenta tomara una serie de medidas para mejorar la forma como desde el gobierno se trataba el tema indígena. La pregunta central que se planteó en ese momento al interior del gobierno decía relación con cómo entender cuáles eran las demandas básicas no resueltas en las comunidades indígenas que estaban latentes y que motivaban el descontento manifestado. Como respuesta aparecían tres cuestiones relevantes, que eran la demanda por derechos políticos, la demanda territorial por tierras y la protección frente a los proyectos de inversión que se iban a realizar en territorios indígenas.

En el gobierno se asumió que estas cuestiones eran los tres grandes ejes que estaban como trasfondo en la generación del conflicto. Frente a eso, el gobierno decidió empezar a preocuparse con mayor intensidad de la situación ya que claramente, de no hacerse nada, podía transformarse en algo fuera de control. Es en este contexto en el cual se dan dos hechos de gran gravedad que marcan una inflexión en el actuar del gobierno. Por una parte el lamentable asesinato del joven activista Matías Catrileo en una ocupación de un fundo reclamado por una comunidad en la zona de Vilcún, Región de la Araucanía, y por otra parte, la huelga de hambre que mantenía una dirigente mapuche en una cárcel de la región.

Frente a lo anterior, al interior del gobierno se instaló la convicción de que había que enfrentar el tema indígena desde una perspectiva de mayor plazo, ya que lo que se había hecho en el pasado, si bien era positivo, reflejaba una visión de corto plazo y que no se hacía cargo del carácter estructural de los tres elementos antes citados, como tampoco se hacía cargo de la conflictividad permanente que se desarrollaba en ciertos territorios. Producto de lo anterior, se tomaron una serie de medidas como son la constitución de un comité especial de ministros encargado del tema, se nominó una autoridad política especial sobre la materia como comisionado presidencial, y se definieron cuatro ámbitos de trabajo como los ejes prioritarios para reordenar el accionar del gobierno. Estos ámbitos eran la recuperación del diálogo entre el gobierno y la dirigencia indígena, la revisión 
de las políticas públicas en la perspectiva de la pertinencia indígena, el empezar a plantear los temas de la multiculturalidad en la sociedad chilena y el avanzar en el tema de reconocimiento constitucional.

A partir de lo anterior se encara una reformulación de la política gubernamental para los pueblos indígenas, la que se enmarca en la perspectiva que daba el hecho de que el Convenio 169 iba a ser ratificado y que posteriormente iba a entrar en vigencia.

\subsection{LA REFORMULACIÓN DE LA POLÍTICA INDÍGENA Y SU ADECUACIÓN A LA ENTRADA EN VIGENCIA DEL CONVENIO}

En el programa de gobierno de la candidatura de Michelle Bachelet se formula un conjunto de objetivos que se intentaría alcanzar en política indígena. En este contexto, la Presidenta enunció en abril del 2007 una agenda de trabajo en materia de política indígena, que identificaba los principales ámbitos del trabajo que se priorizarían. ${ }^{12}$ Pero el avance en la implementación de esta política y los conflictos que se estaban desarrollando, hacían que la evaluación que se tenía al nivel presidencial sobre la acción gubernamental en esta materia era negativa. En este contexto, la Presidenta deseaba impulsar un cambio en la política, tanto en el ritmo que llevaba la implementación de diversos programas como en el enfoque que había guiado la definición de la política hasta ese momento. El nuevo enfoque se expresaba básicamente en los siguientes aspectos:

- en el pasado se había considerado el tema indígena como una cuestión de pobreza y marginalidad; esta mirada se intentaba cambiarla por un enfoque que llevaba a considerarlo un asunto referido a los derechos de los pueblos indígenas.

- desde la vuelta a la democracia se había considerado que la resolución de los temas que afectaban a los pueblos indígenas era un asunto exclusivamente concerniente al gobierno; ahora en cambio, se planteaba tratarlo como un tema en el cual el conjunto de la sociedad debía asumirlo.

12 Nuevos ejes de la Política Indígena. 30 abril 2007. Mideplán. 
- con la promulgación de la Ley Indígena y la creación de la CONADI, se había intentado centralizar en una repartición del gobierno los temas indígenas; el nuevo enfoque buscaba transversalizarlo e institucionalizarlo en toda la estructura estatal.

- por último se proponía avanzar en la construcción de la multiculturalidad, entendida como el respeto a la diferencia entre ciudadanos con igualdad de derechos, en el marco de una sociedad democrática en la que varias culturas se dan cita en un espacio físico común.

Este nuevo enfoque se expresó en la política "Reconocer: pacto social por la multiculturalidad"13 que refleja un análisis de los avances logrados hasta ese momento en la política que había planteado el gobierno en abril del 2007; resume una crítica evaluación sobre lo que se había realizado por los gobiernos precedentes; en función de dicha evaluación, se identificaban los desafíos a enfrentar; a continuación se definen los objetivos a alcanzar para hacerse cargo de los desafíos identificados; de ahí se proponía un Plan de Acción que contenía los diversos ámbitos en los cuales se proponía actuar; y por último se presentaba un "esquema de gobernabilidad" necesario de construir para darle viabilidad política y social a la política.

Es relevante resumir los componentes del Plan de Acción que se estructuró en torno a tres grandes áreas:

1. Sistema Político, derechos e institucionalidad

1.1. Sistema político y derechos indígenas.

1.2. Institucionalidad y políticas públicas.

2. Desarrollo Integral de los pueblos

2.1. Tierras y territorio.

2.2. Desarrollo económico y productivo.

2.3. Educación, salud y cultura.

2.4. Especificidad de los pueblos indígenas.

13 Ver Mideplán, Política "Reconocer: Pacto Social por la multiculturalidad”, 1 de abril 2008. 
3. Multiculturalidad y diversidad

3.1. Política Indígena Urbana.

3.2. Multiculturalidad en la sociedad.

\subsection{GESTIÓN DEL DESARROLLO INTEGRADO}

La puesta en marcha de la nueva política indígena "Reconocer" requería un conjunto de medidas que facilitaran su implementación. En este sentido era necesario desarrollar un esquema de gobernabilidad que permitiera alcanzar el propuesto Pacto Social por la Multiculturalidad; se requería que la sociedad en su conjunto participara del esfuerzo propuesto, destacándose una especial responsabilidad que debían asumir los medios de comunicación; por su parte el Gobierno comprometía el máximo respaldo a esta iniciativa y aseguraba la participación en foros y organismos internacionales; en seguida se señalaba que una de las preocupaciones principales era la solución de los conflictos entre indígenas, comunidades y otros actores privados y entes públicos, siendo en este sentido muy relevante el sistema de certificación de conducta responsable para los proyectos de inversión en tierras indígenas; frente a los conflictos, el Gobierno señalaba que actuaría preventivamente, adelantándose en todo lo posible a los mismos e instalando el diálogo; y por último se planteaba que se favorecerían los cambios necesarios para que la sociedad chilena asumiera plenamente su carácter multicultural.

\section{La propuesta de implementación del Convenio i 69}

La propuesta de implementación del Convenio 169 se enmarca dentro de la política "Reconocer", que es el marco de orientación que usaría el gobierno para plantear la puesta en vigencia del convenio. Por lo tanto, al hacer el análisis que se presenta a continuación, se hará una y otra vez referencia a las partes de la política "Reconocer" que se hacen cargo de las disposiciones del convenio. Ciertamente la forma como se construyó la política no sigue necesariamente el orden de acuerdo al cual se presentan las diversas materias en el convenio; pero sí se puede decir que en el documento de política se asumen casi todas las disposiciones que son parte del convenio. 


\subsection{ASPECTOS GENERALES DE APLICACIÓN}

Los aspectos generales de aplicación dicen relación con la distinción entre normas auto-ejecutables, es decir que iban a ser exigibles desde la entrada en vigencia del convenio, normas programáticas, que no eran auto-ejecutables, que entregaban orientaciones a ser cumplidas en el futuro y que para que se transformaran en normas obligatorias debía hacerse un ajuste en la norma legal específica, y normas que imponían condiciones al accionar del gobierno, como por ejemplo el no atentar contra los derechos humanos o el proteger los derechos laborales, y que por lo tanto imponían al gobierno la obligación de evaluar la forma como dichas normas se estaban aplicando.

La otra cuestión general está relacionada con el asumir que había flexibilidad en la aplicación y adecuación a las circunstancias para determinar el alcance y la naturaleza de las medidas en cada país ${ }^{14}$. Si bien este principio general está claramente estipulado en el texto del Convenio, la forma de interpretarlo puede ser diversa, porque dicha interpretación estará ciertamente influenciada por la manera como cada sector o interesado entienda las circunstancias del país que llevarían a determinar el alcance y naturaleza de las medidas.

\subsubsection{ELEMENTOS A CONSIDERAR EN LA IMPLEMENTACIÓN}

En relación a que se partía de la consideración que las materias previstas en el convenio tenían una determinada regulación a nivel nacional, el punto principal a evaluar era qué normas de las existentes cumplían los estándares exigidos por el convenio y cuáles no lo hacían.

Si se consideraba que una norma cumplía el estándar, lo que se debía hacer era revisar cómo se estaba cumpliendo la norma y si su aplicación imponía o no obstáculos a los pueblos indígenas; en caso que estos obstáculos existieran, era necesario determinar los ajustes necesarios de aprobar.

En cambio si la norma no cumplía con los estándares exigidos por el convenio, esta norma debía someterse a ajustes o perfeccionamientos, ya sea por medio

14 Cf. Art. 34 del Convenio 169. 
de una revisión de la normativa o su derogación y reemplazo por otra que sí cumpliera con dichos estándares.

Demás está decir que frente a esta distinción, nuevamente se abrían posibles diferencias en la evaluación de la materia, entre los diversos actores interesados en el tema.

\subsubsection{EJES DE IMPLEMENTACIÓN DEL CONVENIO}

Para llevar adelante la discusión sobre la implementación, se propuso definir cuatro grandes ámbitos o ejes en torno a los cuales era posible ordenar el trabajo. El primero era el referido a las adecuaciones institucionales básicas que eran necesarias de implementar. El segundo se refería a las adecuaciones normativas. El tercer eje era la revisión de políticas, planes y programas conforme a la pertinencia indígena. Y el cuarto eje decía relación con los asuntos que había que enfrentar en relación con la difusión del convenio y con el involucramiento de otros órganos estatales distintos del gobierno, que pudieran ser relevantes.

\subsection{LAS ADECUACIONES INSTITUCIONALES}

Del análisis del texto del convenio, surgen una serie de asuntos vinculados a la institucionalidad pública que se debiera tener en el país para que el convenio se estuviera cumpliendo.

Una primera disposición es la referida al derecho a definir sus propias prioridades de desarrollo (art. 6.1.b.). Analizando la institucionalidad existente en el país, se constataba que la única instancia de participación y representatividad de los pueblos indígenas era el Consejo de la CONADI, en el cual hay, bajo una presidencia del Director Ejecutivo de la corporación, una membresía paritaria entre dirigentes indígenas y representantes del gobierno. Ciertamente esta fórmula institucional no aseguraba el cumplimiento del estándar definido por el convenio. De ahí que el Reconocer planteaba la creación de un Consejo de Pueblos Indígenas, con personería pública y autónoma del poder ejecutivo, constituido por miembros elegidos directamente por los pueblos.

Una segunda disposición del convenio dice relación con la participación de representantes de los pueblos indígenas en las instituciones electivas y administrativas del país. Condición necesaria para establecer esta participación era 
el reconocimiento constitucional de los pueblos indígenas, reforma que se proponía como absolutamente necesaria de ser aprobada para encaminarse al cumplimiento de esta disposición. Una vez establecido el reconocimiento constitucional en su calidad de pueblos, era posible entrar a pensar formas de representación propia de los pueblos en las instituciones de la democracia representativa y en la administración.

Para cumplir con estos estándares, en el Reconocer se plantean tres niveles de participación. La primera, al nivel del parlamento, era introducir una cierta cantidad de parlamentarios elegidos directamente por el mundo indígena; pensando que la población indígena es el $8 \%$ de la población nacional, se proponía que se eligieran entre 8 a 10 diputados, por sobre la actual cantidad de 120 diputados. En segundo lugar, en los consejos regionales se planteaba algo similar, es decir, tener una representación indígena elegida por los indígenas de la región, en aquellas regiones en las cuales la presencia de población indígena fuera relevante.

En tercer lugar, si bien en el documento Reconocer se planteaba también que hubiese representación indígena directa a nivel de los Concejos Municipales, esta propuesta fue finalmente desechada dado que en las comunas de mayor presencia indígena, y dado el sistema proporcional de elección de concejales, esta presencia ya era posible. La tercera modificación institucional dice relación con lo dispuesto en el art. 2 del convenio que señala que los gobiernos van a tener una acción coordinada y sistemática para allanar el ejercicio de los derechos indígenas. Aquí se hacía una apelación a la estructura del gobierno en su institucionalidad y en la forma.

Para responder a estas disposiciones se decidió en primer lugar la creación por decreto presidencial de un Consejo de Ministros de Asuntos Indígenas, recibiendo este consejo el mandato de definir las políticas de gobierno frente a los temas indígenas.

En segundo término, se decidió crear, al nivel del Gabinete Ministerial, en la forma de una Subsecretaría, una instancia política-administrativa encargada de los asuntos indígenas. A nivel regional, se determinó instalar mesas regionales indígenas, en las ocho regiones donde el tema indígena era más prioritario, así como se determinó la creación, en cada ministerio y en cada gobierno regional, una Unidad de Asuntos Indígenas. 
Con la finalidad de conocer el esfuerzo gubernamental en los temas indígenas en lo presupuestario, se determinó construir una visión de presupuesto indígena integrado, en el cual se reflejaran todas las partidas presupuestarias que tenían como grupo destinatario de las mismas al mundo indígena.

Por último, a nivel institucional, se proponía hacer una transformación de la institucionalidad pública indígena. Teniendo en consideración que se creaba el Consejo de Pueblos Indígenas como ente de representación, que se creaba la Subsecretaría de Asuntos Indígenas como ente de definición de la política pública en la materia, la CONADI debía transformarse en un servicio implementador de las políticas, pasándose así a un modelo en el cual las políticas serían propuestas por la Subsecretaría, consultadas con el Consejo de Pueblos e implementadas por la CONADI.

\subsection{LAS ADECUACIONES NORMATIVAS}

A partir de la entrada en vigencia del Convenio 169, era necesario revisar, y en caso necesario ajustar, un conjunto de leyes y normas para adecuarlas a los estándares exigidos por el convenio. No es posible tratar en este artículo todos y cada uno de los derechos establecidos en el Convenio 169 y revisar el tipo de adecuación normativa que era necesario emprender ${ }^{15}$.

En todo caso es relevante consignar las principales modificaciones que eran necesarias de introducir en el ordenamiento jurídico nacional para adecuarlo a los estándares exigidos por el convenio. Estas son las siguientes:

En el ámbito de los derechos:

a) Derechos humanos / no discriminación: El convenio plantea claramente un conjunto de derechos que deberían ser respetados, entre los cuales está el goce de derechos humanos y libertades fundamentales, la protección contra la violación de los derechos y el poder iniciar procedimientos para asegurar el respeto efectivo de dichos derechos. ${ }^{16}$ Frente a este tema, el Reconocer contemplaba el reconocimiento constitucional de los pueblos

15 Sobre un tratamiento detallado de este tema ver "Convenio 169 sobre pueblos indígenas y tribales en países independientes y su implementación en Chile”, CONADI, marzo de 2009.

16 Art. 3 y 12 del convenio 169. 
indígenas que se inserta en el derecho al reconocimiento de su especificidad y derechos colectivos, y el dar prioridad a la tramitación del proyecto de Ley sobre Discriminación.

b) Derecho a decidir sus propias prioridades en lo que atañe al proceso de desarrollo, en la medida en que éste afecte a sus vidas, creencias, instituciones y bienestar espiritual y a las tierras que ocupan o utilizan de alguna manera, y derecho a controlar, en la medida de lo posible, su propio desarrollo económico, social y cultural ${ }^{17}$. Frente a este derecho, el Reconocer contemplaba la creación del Consejo Nacional de Pueblos Indígenas, el que tendría participación en el diseño y formulación de la política nacional indígena y de políticas públicas que se orientasen al cumplimiento de su objetivo; este Consejo sería la instancia por medio de la cual se contaría con un órgano que hiciera realidad el ejercicio de este derecho, sin perjuicio de mantener la consulta directa a los pueblos indígenas cuando pudiesen ser afectados por medidas específicas.

c) Derecho a participar en formulación, aplicación y evaluación de leyes, planes y programas. ${ }^{18}$ Para implementar este derecho el Reconocer contemplaba la institucionalización del derecho a participación en la definición de leyes, políticas y programas que afectasen a los pueblos indígenas. Asimismo, se preveía el reconocimiento y la operacionalización del derecho a participar en la gestión de las Áreas de Desarrollo Indígena. Además, tratándose de una norma que tendría aplicación desde la entrada en vigencia del Convenio, se debía establecer un procedimiento a través del cual pudiera ejercer este derecho de participación, lo que obligaba a analizar el sistema de planificación, lo que era particularmente relevante en los casos de las regiones con alta concentración de población indígena.

d) Conservar sus costumbres e instituciones propias, siempre que éstas no sean incompatibles con los derechos fundamentales definidos por el sistema jurídico nacional ni con los derechos humanos internacionalmente

17 Artículo 7 No 1 del Convenio, primera parte.

18 Artículo 7 No 1 del Convenio, segunda parte. 
reconocidos ${ }^{19}$, y sin que ello les impida ejercer los derechos reconocidos a todos los ciudadanos del país y asumir las obligaciones correspondientes. ${ }^{20}$

Frente a esta materia, el Reconocer planteaba que en la Ley Indígena ya se contemplaba la protección y promoción del desarrollo de las culturas indígenas así como el derecho a mantener y desarrollar sus propias manifestaciones culturales, estableciéndose que el reconocimiento de una misma jefatura tradicional constituye un criterio para acreditar la pertenencia a una misma comunidad indígena. Asimismo, en la Ley se reconoce la costumbre como derecho a ser invocado entre personas del mismo pueblo indígena y en materia penal, para eximir o atenuar la responsabilidad.

En el ámbito de deberes generales del Estado, las principales obligaciones a cumplir son las siguientes:

a) En la protección de derechos indígenas se debe desarrollar, con la participación de los pueblos indígenas, una acción coordinada y sistemática para proteger sus derechos y garantizar el respeto de su integridad... ${ }^{21}$.

El Reconocer contemplaba la creación de un Consejo de Ministros, que asesoraría al Gobierno en la dirección coordinada en materias indígenas; la creación de las unidades de asuntos indígenas en los Ministerios e Intendencias; el seguimiento de agendas regionales a través de mesas conformadas a ese nivel territorial; y la identificación y gestión integrada de las partidas presupuestarias destinadas a asuntos indígenas a nivel nacional. Además el Reconocer contemplaba la preparación de una propuesta de desarrollo integral de las economías de los pueblos indígenas, fundada en las áreas de desarrollo indígena, protegiendo los recursos naturales, fomentando energías alternativas y tecnologías adecuadas y custodiando la calidad y usos del agua, así como otras medidas en conectividad rural, revisión de políticas sectoriales según pertinencia indígena y especial atención al tema de equidad de género en el contexto de la política indígena.

Además el Estado, según el convenio, deberá adoptar medidas especiales para salvaguardar las personas, instituciones, bienes, trabajo, culturas y medio ambiente

\footnotetext{
19 Artículo 8 No 2 primera parte.

20 Artículo 8 No 3 del Convenio.

21 Artículo 2 del Convenio.
} 
de los pueblos indígenas, sin que contradigan los deseos expresados de los pueblos indígenas. $^{22}$

Siendo este uno de los derechos que estaban más demandados de protección por los pueblos indígenas, el Reconocer contemplaba la elaboración de un Código de Conducta Responsable que permitiera adicionar a la evaluación de los impactos ambientales de los proyectos productivos, los efectos que ellos pudieran tener sobre las culturas indígenas, en términos de afectar bienes inmateriales o valores culturales cuyo conocimiento y ponderación no encontraba un fundamento normativo claro.

b) En la aplicación del Convenio el Estado debería reconocer y proteger los valores y prácticas sociales, culturales, religiosas y espirituales propias de dichos pueblos, así como la integridad de sus valores, prácticas e instituciones y tomar debidamente en consideración la índole de los problemas que se les plantean tanto colectiva como individualmente. Respetar, asimismo, la integridad de los valores, prácticas e instituciones de esos pueblos ${ }^{23}$. Frente a este derecho, el Reconocer contempla el reconocimiento de las prácticas culturales, sociales, deportivas y religiosas, sin ser más explícito sobre la forma como esto se concretaría.

c) Así mismo el convenio estipula que se deberá implementar el derecho a consulta. El convenio establece la obligación de los gobiernos a consultar a los pueblos indígenas, mediante procedimientos apropiados y, en particular, a través de sus instituciones representativas, cada vez que se prevean medidas legislativas o administrativas susceptibles de afectarles directamente. Dicha consulta debería efectuarse de buena fe y de una manera apropiada a las circunstancias, con la finalidad de llegar a un acuerdo o lograr el consentimiento acerca de las medidas propuestas..$^{24}$

Esta norma fue considerada como auto-ejecutable por el Tribunal Constitucional ${ }^{25}$. Asimismo, estimó que modificaba leyes orgánicas constitucionales (Congreso Nacional, Bases Generales de la Administración del Estado, Gobiernos Regionales

22 Artículo 4 del Convenio.

23 Artículo 5 a) y b) del Convenio.

24 Artículo 6 No 1 a) y No 2.

25 Considerando $7^{\circ}$, sentencia Rol No 309. 
y Municipalidades) desde el momento que hacía obligatorio efectuar una consulta dentro de los procedimientos llevados adelante por diversos órganos.

El Reconocer asumía este ajuste normativo, desde un punto de vista de la gestión de gobierno, en los procedimientos instalados por medio del Instructivo Presidencial Nr. 5 ya citado, así como en la creación de las Unidades de Asuntos Indígenas en los ministerios e intendencias. Además en relación a la instalación de la consulta como una práctica obligatoria en el Estado, el Reconocer señalaba que era necesario dictar una norma clara en la materia, asunto que en su fase de ejecución se complicó sobremanera al ser dictado el Decreto nr. 24 -2009 de Mideplán. ${ }^{26}$

d) En adición, el Estado debía establecer los medios para el pleno desarrollo de las instituciones e iniciativas de esos pueblos, y en los casos apropiados proporcionar los recursos necesarios para este fin. En relación a este tema, el Reconocer contemplaba la creación del Consejo Nacional de Pueblos Indígenas, financiado con recursos públicos, a través del cual se representaría a los pueblos indígenas en el diseño y seguimiento de la política nacional indígena. Por último, debe destacarse la obligación referida a velar porque, siempre que haya lugar, se efectuasen estudios, en cooperación con los pueblos indígenas, a fin de evaluar la incidencia social, espiritual y cultural y sobre el medio ambiente que las actividades de desarrollo previstas pudiesen tener sobre esos pueblos. ${ }^{27}$ Asimismo, el convenio señala que se deben tomar medidas, en cooperación con los pueblos interesados, para proteger y preservar el medio ambiente de los territorios que habitan.

Estas obligaciones eran asumidas por el Reconocer en la propuesta de elaboración de un Código de Conducta Responsable mediante el cual se pretendía instaurar un procedimiento que permitiera establecer, ponderar y adoptar medidas que evitaran o mitigaran los impactos que los proyectos productivos ocasionasen a las culturas indígenas, planteando que en el ámbito medioambiental, continuara operando el Sistema de Evaluación de Impacto Ambiental SEIA.

En el ámbito de las normas referidas a las tierras, se pueden destacar los siguientes aspectos:

26 Ver Decreto nr. 24 del 2009, Mideplán.

27 Artículo 7 No 3 del Convenio. 
a) Derechos de los pueblos indígenas a los recursos naturales existentes en sus tierras. Estos derechos comprenden el derecho de esos pueblos a participar en la utilización, administración y conservación de dichos recursos ${ }^{28}$. Dada la variedad de textos legales existentes que tenían una relación con la implementación de este derecho, el Reconocer contemplaba como iniciativa el reconocimiento y la expresión en nuevos ordenamientos legales del derecho sobre recursos naturales en las Áreas de Desarrollo Indígenas y en las tierras indígenas (en especial aguas, geotermia, minas y pesca). Al mismo tiempo estaba claro que el sistema de participación en el marco de los estudios de impacto ambiental definidos en la Ley del Medio Ambiente debía ser revisado a fin de determinar su adecuación a la participación exigida en el Convenio 169, ya que el procedimiento previsto por el SEIA no contenía especificidades que se hicieran cargo de contemplar mecanismos apropiados con pertinencia indígena.

b) El convenio señalaba también que en caso de que perteneciesen al Estado la propiedad de los minerales o de los recursos del subsuelo, o que tuviera derechos sobre otros recursos existentes en las tierras, se debía respetar el derecho a participar siempre que sea posible en los beneficios que reporten tales actividades, y percibir una indemnización equitativa por cualquier daño que puedan sufrir como resultado de esas actividades ${ }^{29}$.

Ciertamente esta disposición del convenio es una de las que mostraba mayores implicancias desde el punto de vista de las adecuaciones normativas. El Reconocer proponía responder de forma integral a este derecho en el ya mencionado Código de Conducta Responsable, así como en la revisión de la normativa sectorial en materias de minería, geotermia y aguas.

c) Además se establece el derecho a no ser trasladados de las tierras que ocupan, salvo $^{30}$ que excepcionalmente el traslado y la reubicación se consideren necesarios, y sólo deberán efectuarse con su consentimiento, dado libremente y con pleno conocimiento de causa. Además se establece el derecho de regresar a sus tierras tradicionales en cuanto dejen de existir las causas que motivaron

28 Artículo 15 No 1 del Convenio.

29 Artículo 15 № 2 del Convenio.

30 Artículo 16 del Convenio. 
su traslado y reubicación, siempre que sea posible, el derecho a recibir tierras cuya calidad y estatuto jurídico sean iguales a los de las tierras que ocupaban o si prefirieran una indemnización. Todo esto debería realizarse por acuerdo o por medio de procedimientos adecuados. Este derecho no se encuentra consagrado en nuestra legislación y, en cualquier caso, es más amplio que el derecho de propiedad. Este derecho de no traslado y los consiguientes se contemplarían en el Código de Conducta Responsable.

d) El convenio establece que será un deber del Estado en materia de tierras respetar la importancia especial que para las culturas y valores espirituales de los pueblos indígenas reviste su relación con las tierras o territorios ${ }^{31}$, reconocer a los pueblos indígenas el derecho de propiedad y de posesión sobre las tierras que tradicionalmente ocupan ${ }^{32}$, y otras medidas referidas al derecho a utilizar las tierras ocupadas por ellos, al garantizar la protección efectiva de sus derechos de propiedad, y a resolver las reivindicaciones de tierras formuladas por los pueblos.

El Reconocer contemplaba como iniciativas la entrega de tierras a 115 comunidades priorizadas por el Consejo de CONADI; la resolución de las solicitudes de tierras de 308 comunidades, cuya aplicabilidad se definirá en el periodo 2008-2010, a partir de lo cual se preparará un plan quinquenal a partir de 2011 para resolver lo que se priorizaría; la manutención y aumento del programa de tierras a través de subsidios; y, paralelamente, la evaluación del actual mecanismo de asignación; y la mejora en la gestión del Fondo de Tierras y Aguas de CONADI, precisando la política de tierras de CONADI y aclarando los criterios para la aplicación de la Ley.

\subsection{LA REVISIÓN DE POLÍTICAS, PLANES Y PROGRAMAS CONFORME A LA PERTINENCIA INDÍGENA}

Un conjunto de disposiciones del convenio debían enfrentarse más bien con medidas de gestión, que apuntaran a establecer en qué medida las políticas, planes y programas en implementación cumplían los estándares definidos por el convenio; en la medida que no los cumplieran, deberían generarse adecuaciones

\footnotetext{
31 Artículo 13 No 1 del Convenio.

32 Artículo 14 No 1 del Convenio.
} 
en dichas materias o en su caso proponer nuevas normas legales. Estas materias se referían principalmente a las condiciones de contratación y empleo, a la formación profesional, artesanía e industria rural, a la Seguridad Social y salud, a la educación y medios de comunicación.

Frente a ellas el Reconocer proponía una diversidad de medidas que intentaban responder en las respectivas políticas públicas a los estándares exigidos por el convenio.

En materias de empleo el Reconocer no contempla iniciativas específicas, por lo cual se planteaba como necesidad realizar un primer trabajo de elaboración del diagnóstico de la situación, al que concurrieran los diversos organismos con competencia en la materia y con la colaboración de los pueblos indígenas.

En relación a lo dispuesto en materias de Formación profesional, artesanía e industria rural, el Reconocer contemplaba la preparación de una propuesta de desarrollo integral de las economías de los pueblos indígenas y, el programa de innovación cuyo objetivo era la incorporación de las economías indígenas a la dinámica de los clusters productivos.

En lo relativo a Salud y Seguridad Social, la política Reconocer contemplaba el reforzamiento del Programa Especial de Salud de Pueblos Indígenas del Ministerio de Salud, con especial atención a la recuperación de la biodiversidad y la medicina indígena. Asimismo, se contemplaba en el proyecto de ley de los derechos de los pacientes, el otorgamiento de prestaciones de salud con carácter intercultural.

En lo relacionado con Educación y Medios de Comunicación el Reconocer contemplaba la ampliación de la cobertura del Programa de Becas Indígenas y la definición, en un proceso participativo, del programa de hogares indígenas. Así mismo se contemplaba reconocer en la Ley General de Educación el derecho de los pueblos a incidir en la educación de sus miembros y el impulso al programa de educación intercultural en jardines infantiles y escuelas de alta presencia indígena desarrollado por el Ministerio de Educación. Además se definía impulsar la enseñanza de las lenguas indígenas y la incorporación de formadores tradicionales. Asimismo, se contemplaba la implementación de iniciativas destinadas a fomentar la lengua y la cultura indígenas, como la creación de academias de lenguas indígenas y el potenciamiento de al menos cuatro institutos de cultura indígena en las universidades. 
En relación con la multiculturalidad, el Reconocer contemplaba la difusión del Informe de Verdad Histórica y Nuevo Trato en todos los establecimientos educacionales del país; modificaciones curriculares para plasmar en contenidos educativos y en la enseñanza escolar de la historia nacional la visión del informe antes señalado; y la construcción de una política de multiculturalidad y otra de inclusión de las diversas culturas.

Por último proponía el reconocimiento de las prácticas culturales, sociales, deportivas y religiosas, contemplándose entre las iniciativas legales la transmisión matrilineal de apellidos y la anotación en la inscripción de nacimiento de la localidad de origen.

\subsection{LA DIFUSIÓN DEL CONVENIO Y EL INVOLUCRAMIENTO DE OTROS} ÓRGANOS ESTATALES RELEVANTES

El cuarto aspecto que contemplaba la propuesta de implementación del convenio decía relación con la difusión del mismo en el país y con las responsabilidades que les tocaba asumir a otros órganos del Estado. Una primera materia a ser trabajada está vinculada a la forma como se garantizarían los derechos humanos que se establecen en virtud del convenio. Esto hacía necesario el perfeccionamiento de los sistemas de asistencia jurídica existentes y la aplicación de orientaciones apropiadas en materia penal en el Ministerio Público, en la Defensoría Penal Pública y en el Poder Judicial.

Así mismo aparecería necesario perfeccionar la forma como estos derechos pasarían a ser defendidos frente a la administración del Estado; en este sentido tomaba un rol particular la Comisión de Defensa de los Derechos Ciudadanos, que si bien tiene un poder restringido es el único órgano de la administración que tiene encomendada dicha tarea.

Un segundo asunto a ser tratado era el relacionado con la forma como el gobierno debía asumir la difusión de los derechos políticos, sociales, laborales y otros, que se establecían en virtud del convenio. De poco valía que estos derechos fueran ley por la vigencia legal del convenio si no eran conocidos por sus destinatarios. Lo anterior obligaba a redefinir el trabajo del Programa de Información sobre Derechos Indígenas (PIDI) de la CONADI y a generar programas especiales que asumieran esta tarea. 
Una tercera materia que debía ser trabajada era la forma como otros órganos del Estado, distintos del poder ejecutivo, debían implementar la obligación de la consulta. Esto aplicaba principalmente al Congreso Nacional y a las Municipalidades, ya que las disposiciones que el gobierno pudiera definir para la realización de la consulta indígena, no obligaría a estos órganos constitucionalmente autónomos del ejecutivo.

Por último, la adecuada entrada en vigencia del convenio, planteaba una serie de asuntos que eran atingentes a la comunidad nacional como conjunto. Además de la necesaria difusión del contenido del convenio, era necesario que el tema de los pueblos indígenas pasara a tener un lugar más relevante en la agenda política nacional. A este fin, el Reconocer planteaba diversas medidas ya mencionadas como la publicación del Informe de la Comisión Verdad Histórica y Nuevo Trato, las modificaciones curriculares en los planes de enseñanza, la construcción de una política de multiculturalidad y otras medidas.

\section{LA CONSULTA INDÍGENA Y LA DISCUSIÓN SOBRE CÓMO IMPLEMENTAR el Convenio i69}

La primera materia que debía ponerse en ejecución al momento de la entrada en vigencia del convenio era la consulta indígena. Como se ha señalado en párrafos anteriores, esta materia había sido declarada como auto-ejecutable por el Tribunal Constitucional, es decir, entraba a ser exigible desde el momento en que el convenio entrara en vigencia.

Por esta razón, junto con preparar la propuesta de implementación que ha sido reseñada en el capítulo tres de este artículo, se trabajó una propuesta de implementación de la consulta.

El tener que normar la consulta ya significaba poner un tema nuevo en la agenda pública; hasta ese momento no se había aprobado una ley sobre participación ciudadana en la gestión pública ni tampoco había sido reglamentado el sistema de participación indígena en la ley respectiva.

En general los directivos públicos habían sido reacios a la implantación de programas amplios de participación ciudadana y estos sólo habían podido avanzar 
producto de la dictación de diversos instructivos presidenciales sobre la materia, tanto en los gobiernos del Presidente Ricardo Lagos como en el de la Presidenta Michelle Bachelet. ${ }^{33}$

En el período que se tuvo entre la ratificación del convenio y su puesta en marcha se realizaron un conjunto de consultas a modo experimental. Es así como se realizó una consulta sobre la reforma constitucional de reconocimiento de los pueblos indígenas, otra sobre las propuestas de creación del Consejo de Pueblos Indígenas y la participación indígena en el parlamento, así como consultas particulares sobre la posibilidad de sacar un moai de la Isla de Pascua y la creación de un parque nacional en el Salar de Atacama. Todas estas consultas se realizaron sin contar aún con una norma legal que les diera sustento, sino que solamente basadas en el Instructivo Presidencial Nr. 5 de junio del 2008 que señalaba éste como un compromiso asumido por el gobierno Bachelet.

Sin pretender entregar una visión completa sobre el complejo tema de la consulta indígena, deseo presentar la forma como se concibió la puesta en funcionamiento de la consulta a partir de la entrada en vigencia del convenio.

Los fundamentos normativos que sustentaban la consulta eran el artículo 6 del Convenio 169 de la OIT, que establece que se deberá consultar a los pueblos indígenas mediante procedimientos apropiados, a través de sus instituciones representativas, cada vez que se prevean medidas legislativas o administrativas susceptibles de afectarles directamente. Además el artículo 7 establece que deberán participar en la formulación, aplicación y evaluación de los planes y programas de desarrollo nacional y regional susceptibles de afectarles directamente.

Por último el artículo 6 en sus números 1 y 2, y el artículo 7 número 1 oración segunda del Convenio 169 eran normas auto-ejecutables sin tener carácter vinculante, en virtud de lo que había señalado el Tribunal Constitucional.

Los principios a ser respetados en la implementación de la consulta eran su carácter previo, que la consulta no se agota en la información, que debía hacerse de buena fe, que debía respetarse la representatividad, hacerse usando

33 Ver "Instructivo Presidencial sobre Participación Ciudadana” del Presidente Ricardo Lagos del 7 de diciembre de 2000 y "Agenda pro participación ciudadana" del Gobierno de Michelle Bachelet, del 2008. 
procedimientos adecuados y buscando el acuerdo y el consentimiento previo de los afectados.

La consulta tenía ciertos objetivos que eran conocer, recoger e incorporar visiones, intereses y propuestas de los pueblos y/o comunidades indígenas interesadas en el diseño de las medidas legislativas o administrativas que les estén dirigidas y/o puedan afectarles directamente; y recoger sus opiniones sobre la pertinencia y conveniencia de implementarlas.

En este contexto, las medidas que debían consultarse eran las siguientes:

a) medidas legislativas, como los proyectos de reforma constitucional, proyectos de ley y reglamentos que afectaren directamente a los pueblos indígenas.

b) medidas administrativas, como las nuevas políticas, planes y programas formulados o elaborados por la administración pública que afectaren directamente a los pueblos indígenas. Además las propuestas de nuevas políticas, planes y programas, debían contemplar mecanismos de participación de los pueblos indígenas en su aplicación y evaluación.

Respecto a qué organismos de la administración debían consultar se señalaba que lo debían hacer todos los órganos de la Administración del Estado, incluyendo gobiernos regionales y municipalidades.

Para determinar qué medidas pueden afectar directamente a los pueblos indígenas, se consideraban los siguientes ámbitos de afectación:

a) aquellos casos en que el elemento territorial estuviera involucrado, por ejemplo, medidas referidas a tierras indígenas, Áreas de Desarrollo Indígena, recursos naturales en unas y otros, sitios arqueológicos, históricos o de importancia ritual, recreativa, etc.

b) aquellos casos en que se afectare directamente grupos humanos indígenas.

Bastaba con que la medida cumpliera una de estas condiciones para que se requiriera consultar. 


\section{LOS ElEMENTOS CRÍTICOS EN LA IMPLEMENTACión DEL CONVENiO}

La implementación de la puesta en marcha del Convenio no estuvo exenta de dificultades. Si habían pasado 18 años antes que el convenio fuese aprobado por el parlamento, no era de esperar que su puesta en funcionamiento fuese algo simple y sin complicaciones. La diversidad de posturas sobre el contenido del convenio y sobre los cambios a introducir en la normativa nacional para adecuarse a los estándares del mismo, no facilitaban una entrada en vigencia del convenio.

Analizaremos un conjunto de ámbitos que a nuestro juicio fueron relevantes en los primeros meses de vigencia del convenio y que se han mantenido como elementos que hacen compleja su aplicación.

\section{La consulta indígena}

El primer tema en torno al cual se estableció una controversia fue el relativo a cómo se normaría la consulta a la que obliga el convenio. Vigente el convenio, cualquier reglamento o ley que normara la forma como se debía hacer la consulta indígena, debía ser consultada. Y ahí se entró en una absurda discusión, sobre si la propuesta de reglamento sobre la consulta que debía preparar el gobierno debía ser puesta en consulta una vez preparada por el gobierno o si ya por el hecho de que el gobierno generaba un proyecto de reglamento, éste estaba no cumpliendo con el convenio, por no haberse consultado la propuesta de proyecto de reglamento. Desde todo punto de vista, la segunda postura impedía a la autoridad iniciar cualquier acción que fuese a ser consultada en el futuro.

El gobierno resolvió proponer un reglamento que normara la consulta, que se refirió a la pendiente reglamentación del art. 34 de la Ley Indígena, que versa sobre la participación indígena. La preparación de este reglamento generó intensas discusiones al interior del gobierno, las que finalmente se plasmaron en el DS 124 de MIDEPLAN del 4 de septiembre del 2009 sobre Consulta y Participación. Este reglamento fue planteado inicialmente como una norma transitoria que fuese utilizada para realizar la "consulta sobre la consulta". Pero surgió la interrogante sobre si lo transitorio no podía pasar a quedar como permanente, sobre todo si venía un cambio de gobierno y posiblemente de coalición, y las nuevas autoridades podían tener concepciones diferentes sobre cómo normar la consulta. 
Y al mismo tiempo, por estar ya al fin del período gubernamental de la Presidenta Bachelet, se constató que no había tiempo suficiente para hacer una consulta sobre este reglamento, que cumpliera con los estándares del convenio. Esto fue el primer escollo en la implementación del convenio 169, que no fue resuelto en forma adecuada.

\section{Reconocimiento constitucional}

El reconocimiento constitucional de los pueblos indígenas estaba puesto en la agenda de demandas indígenas desde el Acuerdo de Nueva Imperial de 1988. Poco se había avanzado en casi 20 años de discusión y el marco de política indígena expresado en el Reconocer ofrecía una perspectiva más positiva para que finalmente esta reforma constitucional fuese aprobada.

El escollo fundamental estaba en la negativa de un sector importante de senadores a reconocer a los indígenas en su condición de "pueblos". Ciertamente si este reconocimiento no se incluía en la reforma, ésta no satisfacería a los dirigentes indígenas y a sus comunidades ni a los defensores de los derechos humanos ni a los dirigentes políticos que estaban comprometidos con el reconocimiento integral y no parcial -sólo como individuos- de los indígenas.

El texto que el Gobierno había logrado aprobar a nivel de la Comisión de Legislación, Constitución y Justicia del Senado, no dejaba a todos contentos, pero era un avance. Este texto fue sometido a consulta indígena, en el período que medió entre la ratificación y la entrada en vigencia del Convenio 169. Y sobre esta consulta se pronunció el Relator de NNUU para Asuntos Indígenas James Anaya.

Si bien la consulta experimental hecha por el gobierno fue implementada y se pusieron en conocimiento de la Comisión del Senado sus resultados, el Senado mismo no llegó a implementar una consulta amplia sobre la reforma que estaban discutiendo; sólo invitó a una veintena de dirigentes y expertos a sesiones de la Comisión. Finalmente la discusión en la sala del Senado no avanzó y nunca llegó a discutirse en sala lo que había sido aprobado por la comisión. Así quedó entrampado el avance en la discusión, con el consiguiente negativo efecto en la dirigencia indígena que vio nuevamente frustrado el intento de llegar a concretar el reconocimiento constitucional. 


\section{Adecuaciones de marcos legales}

En la propuesta de implementación del convenio que había planteado el gobierno, se contemplaba la realización de un estudio jurídico que permitiera clarificar si era necesario realizar adecuaciones en el ordenamiento jurídico para ajustarlos a los estándares del Convenio 169.

En virtud de un acuerdo entre el gobierno y la OIT, ésta contrató a la Universidad Diego Portales para que realizara un informe preliminar sobre la materia.

Dado que los contenidos de este informa fueron muy generales, el gobierno acordó contratar a otras cinco universidades, que teniendo fortalezas en sus facultades de derecho mostraban miradas diferentes sobre la materia, con la finalidad de completar el estudio realizado por la Universidad Diego Portales. En la preparación de este estudio se reflejaron nuevamente las diversas concepciones que existían entre personeros de gobierno, respecto a la necesidad de adecuar o no los marcos normativos existentes.

El hecho de estar ya en los últimos meses de gobierno, llevó a que sólo quedaran preparados los términos de referencia del estudio, pero no se llegó a concretar el encargo, dejando el tema en una situación de nebulosa; por una parte mostraba que, los que en el gobierno estimaban que los cambios legales no eran necesarios, aparecieron en esta materia ganando y por otra, que frente a temas claves como aguas, geotermia, concesiones mineras y eléctricas, y otras asuntos, se mantendría la norma vigente que no respondía a los estándares del convenio.

\section{Regulación de inversiones en tierra indígena}

Siendo esta una de las tres demandas principales del mundo indígena y frente a la cual el Reconocer planteaba una forma de encararla, la manera como se desarrolló durante el año 2009 la discusión sobre la materia dejó la impresión que el gobierno no estaba disponible para entrar a crear un marco regulatorio especial para este asunto. El Reconocer planteaba crear lo que fue denominado en dicho documento "Código de Conducta Responsable" (CCR), que se entendía como el sistema que permitiría realizar la consulta indígena ante proyectos de inversión de acuerdo a los estándares del convenio.

El borrador de CCR fue presentado a la discusión pública en abril del 2009. 
La propuesta generó fuertes reacciones de diversos actores, sobre todo del sector empresarial, que presionó al gobierno para que esta discusión fuese abortada. La dirigencia indígena, si bien reaccionó en forma dividida frente a la propuesta (algunos la rechazaron de plano y otros vieron en ella una posibilidad de disponer de mayor defensa de sus tierras y territorios en el futuro), entendió que la suspensión de la discusión de la propuesta de CCR significaba que sobre esta demanda al menos, en el período del gobierno Bachelet, no se innovaría. Esta situación afectó negativamente en el clima de relaciones entre dirigencia indígena y gobierno.

\section{Adecuación de prácticas intragobierno}

Quizá éste fue el ámbito en el cual se tuvo mayores avances en el programa de implementación del convenio 169. Era comprensible que, existiendo una clara voluntad presidencial expresada tanto en el documento de política Reconocer como en el Instructivo Presidencial del 2008, los ejecutivos de gobierno debían responder a esta orientación presidencial.

Fueron creadas las Unidades de Asuntos Indígenas que significaron un progreso en el tratamiento de los temas indígenas en las instancias de gobierno, empezándose a transversalizar la responsabilidad sobre los mismos al interior del ejecutivo. La mayor dificultad se encontró cuando se tuvo que entrar a precisar qué se entendía por "pertinencia indígena". Mucho se había hablado sobre esta necesidad, pero apareció una nueva y difícil tarea al intentar traducir en términos precisos el significado de la pertinencia en las políticas públicas y en programas en ejecución.

Junto a lo anterior, era previsible que los tiempos de adecuación de programas públicos a las nuevas orientaciones tomaran tiempo. Los nuevos criterios a ser aplicados se plantearon durante el año 2008; no todos alcanzaron a ser tomados en consideración en la preparación del presupuesto del 2009; los que pudieron ser incorporados, obligaron a empezar a rediseñar programas y normativas de aplicación en el primer semestre del 2009; al momento de entrada en vigencia del convenio, quedaba aún trabajo por hacer, lo que se vino a traducir en la ejecución de nuevos programas (como el que se conoció en su momento como el Procesal Indígena del INDAP o el programa de infraestructura de caminos para comunidades del MOP) sólo en el año 2010, ya bajo un nuevo gobierno. 


\section{Los otros poderes del Estado}

Tal como se señaló más arriba en este artículo, el convenio obliga a los Estados que lo ratifican, no solo a los gobiernos de dichos Estados. Pero en el caso chileno, la preocupación principal la había asumido el gobierno. Los municipios o poderes locales, por ser autónomos del gobierno central, no asumieron una responsabilidad en un primer momento. Más aún, las autoridades municipales que estaban más disponibles para entrar a responder a los estándares del convenio, planteaban la necesidad de recibir apoyo financiero adicional del gobierno central para emprender estas tareas.

Por su parte el congreso nacional en un primer momento no propuso mecanismos específicos para asumir esta nueva responsabilidad. Se planteó que los sistemas de invitar a personeros indígenas al debate de las comisiones podían ser suficientes. El congreso no ha avanzado en definir un mecanismo de consulta que sea más amplio e inclusivo de todas las comunidades interesadas en cierta materia.

Por último, el poder judicial no aparecía como una instancia de gobierno que debía implementar mecanismos especiales de consulta. Eso sí, que la entrada en vigencia del Convenio 169 generaba una nueva realidad para el tratamiento de asuntos que involucraran a indígenas o sus comunidades.

\section{A modo de cierre}

El Convenio 169 está plenamente vigente en nuestro país. Esto no significa que no persistan diversas interpretaciones sobre los alcances de las disposiciones del convenio y la forma como debería implementarse.

Le correspondió al gobierno de la Presidenta Michelle Bachelet ratificar y promulgar el convenio como ley de la república y ocuparse de su entrada en vigencia. A este fin, dicho gobierno propuso una forma de implementación del convenio que era amplia y que cubría el conjunto de ámbitos necesarios de ser tratados para lograr una puesta en vigencia que respondiera a lo exigido en el convenio. Lamentablemente el tiempo disponible para alcanzar resultados permanentes fue muy corto; ante materias que eran controvertidas y que existían diversas posturas en el país, no se tuvo la posibilidad de aplicar iniciativas con la persistencia que habría sido requerida. 
$\mathrm{Al}$ producirse el cambio de gobierno en marzo del 2010 y al asumir una coalición de partidos políticos que había tenido posturas refractarias al convenio y a su implementación, la propuesta generada por el gobierno Bachelet fue dejada de lado. En los hechos, no fue sustituida por una propuesta alternativa, sino que se empezaron a aprobar diversas medidas que expresaban una visión distinta sobre la relevancia del convenio, sobre el significado de sus disposiciones y sobre las acciones que le correspondían implementar al gobierno para que el convenio fuese ejecutado correctamente. Por su parte, la dirigencia indígena ha tomado conciencia del valor del Convenio 169 y ha empezado a invocarlo en forma recurrente en la defensa de una diversidad de derechos.

El convenio se ha mantenido en la discusión pública, en los medios de comunicación, en el mundo académico, en los círculos empresariales, en el poder judicial y en menor medida en el mundo parlamentario y político.

La implementación del Convenio 169 es aún, a tres ańos de su entrada en vigencia, una puerta abierta; el cerrarla dependerá de las voluntades políticas que se logren consensuar en la sociedad, que permitan reconocer la existencia de los pueblos indígenas en la plenitud de sus derechos y deberes y que ayuden a avanzar hacia una sociedad multicultural.

\section{Bibliografía}

Aylwin, J. (2008). "Convenio 169 de la OIT y disyuntiva de la Presidenta Bachelet", en El Mostrador, 24 marzo 2008.

“Acuerdo de Nueva Imperial" (1989). 1 de diciembre 1989. En "Hitos de la Política Indígena". 2008.

CONADI, Mideplán (2009). "Convenio nr. 169 sobre pueblos indígenas y tribales en países independientes y su implementación en Chile”.

CONADI, MIDEPLAN (2008). "Hitos de la Política Indígena. 15 años de la Ley Indígena. 1993 - 2008”. Esta recopilación contiene la casi totalidad de bibliografía oficial relevante sobre los temas indígenas, tanto la nacional como la internacional.

Comisionado Presidencial para Asuntos Indígenas (2008). "Informe sobre recomendaciones del relator especial sobre la situación de los derechos humanos y las libertades fundamentales de los indígenas en Chile”. 
Senado (2009). "Informe de la Comisión de Constitución, Legislación, Justicia y Reglamento, recaído en dos proyectos de reforma constitucional, en primer trámite constitucional, sobre reconocimiento de los pueblos indígenas". BOLETINES No 5.324-07 y 5.522-07, refundidos. Valparaíso, 22 de enero de 2009.

IFC, Grupo Banco Mundial (2007). "ILO Convention 169 and the Private Sector. Questions and Answers for IFC Clients".

OIT (2008). "Propuesta de implementación de iniciativas sobre participación de los pueblos indígenas en el sistema político e institucional". Informe interno.

OIT (2008). “Adecuación de la normativa nacional al artículo 15 no 1 del convenio 169 de la OIT, con especial atención a la legislación minera, de energía, de aguas, forestal, de pesca y acuicultura”. Informe interno.

Ley 19.253 del 5 de octubre de 1993, Ley Indígena. En “Hitos de la Política Indígena”. 2008.

"Informe de la Comisión sobre la Verdad Histórica y el Nuevo Trato con los pueblos indígenas", 16 de abril de 2004.

MIDEPLAN (2008). “Reconocer, Pacto social por la multiculturalidad”, 1 de Abril de 2008. 
